\title{
Clinical Usefulness of Dual Red Imaging in Gastric Endoscopic Submucosal Dissection: A Pilot Study
}

\author{
Naoki Yorita', Shiro Oka ${ }^{2}$, Shinji Tanaka ${ }^{1}$, Takahiro Kotachi', Naoko Nagasaki ${ }^{2}$, Kosaku Hata ${ }^{2}$, Kazutaka Kuroki ${ }^{2}$, \\ Kazuhiko Masuda', Mio Kurihara ${ }^{2}$, Mariko Kiso ${ }^{2}$, Tomoyuki Boda ${ }^{1}$, Masanori Ito ${ }^{2}$ and Kazuaki Chayama ${ }^{2}$ \\ Department of ${ }^{1}$ Endoscopy, ${ }^{2}$ Gastroenterology and Metabolism, Hiroshima University Hospital, Hiroshima, Japan
}

Background/Aims: Dual red imaging (DRI) is a new, image-enhanced endoscopy technique. There are few reports about the usefulness of DRI during gastric endoscopic submucosal dissection (ESD). We aimed to examine the usefulness of DRI in endoscopic hemostasis during gastric ESD.

Methods: We enrolled a total of 20 consecutive patients who underwent gastric ESD. Five endoscopists compared DRI with white light imaging (WLI) for the visibility of blood vessels and bleeding points while performing endoscopic hemostasis.

Results: The visibility of blood vessels was increased in 56\% (19/34) of the cases, and the visibility of bleeding points was improved in $55 \%(11 / 20)$ of the cases with the use of DRI compared with the use of WLI.

Conclusions: DRI improved the visibility of blood vessels and bleeding points in cases with oozing bleeding, blood pooling around the bleeding points, and multiple bleeding points. Clin Endosc 2020;53:54-59

Key Words: Dual red imaging; Endoscopic hemostasis; Endoscopic submucosal dissection; Gastrointestinal bleeding; Imageenhanced endoscopy

\section{INTRODUCTION}

Endoscopic hemostasis for gastrointestinal bleeding while performing gastric endoscopic submucosal dissection (ESD) is often difficult with conventional white light imaging (WLI) when there is blood pooling at the bleeding points. Recently, a new image-enhanced endoscopy technique has been developed that consists of 3 types of illumination with wavelengths of 540,600, and $630 \mathrm{~nm}$. Light with 540-nm wavelength can visualize small blood vessels in shallow tissue, whereas light with 600- and 630-nm wavelengths (dual red imaging [DRI])

Received: March 13, 2019 Revised: April 20, 2019

Accepted: May 9, 2019

Correspondence: Shiro Oka

Department of Gastroenterology and Metabolism, Hiroshima University Hospital, 1-2-3 Kasumi, Minami-ku, Hiroshima 734-8551, Japan

Tel: +81-82-257-5191, Fax: +81-82-257-5194, E-mail: oka4683@hiroshima-u.ac.jp ORCID: https://orcid.org/0000-0002-1652-0743

(c) This is an Open Access article distributed under the terms of the Creative Commons Attribution Non-Commercial License (http://creativecommons.org/ licenses/by-nc/3.0) which permits unrestricted non-commercial use, distribution, and reproduction in any medium, provided the original work is properly cited. can penetrate deep tissue. Reflected light at these wavelengths is visualized with little attenuation when there are no blood vessels; however, the reflected $600-\mathrm{nm}$ light is attenuated in the presence of blood vessels in deep tissue. In this technique, images are easily obtained with only a push of a button. Recognizing blood vessels in the submucosal layer is important in endoscopic hemostasis. When bleeding occurs, especially with pooling of blood, it is difficult to find the specific bleeding point. This is because continuous bloodstream around the bleeding vessels obstructs vision during gastric ESD.

The aim of this study was to examine the usefulness of DRI in endoscopic hemostasis during gastric ESD. We conducted a retrospective study to assess the visibility of blood vessels and bleeding points in the submucosal layer with the use of DRI.

\section{MATERIALS AND METHODS}

\section{Patients}

We enrolled a total of 20 consecutive patients who underwent gastric ESD at Hiroshima University Hospital with 
the use of DRI between August 2018 and October 2018. The patients in whom DRI was used were chosen randomly. We retrospectively evaluated the available images and videos to compare DRI with WLI in all patients who underwent gastric ESD. All patients enrolled in this study provided written informed consent for ESD. This study was carried out with approval from the institutional review board and ethics committee of Hiroshima University (no. E-1106) and was in accordance with the World Medical Association Helsinki Declaration.

\section{Procedure of ESD}

ESD was performed using a prototype endoscope (GIF-Y0043; Olympus, Tokyo, Japan). We used a needle knife for precutting and performed circumferential mucosal incision and submucosal dissection with insulated-tip knife 2 and an electrosurgical generator (VIO 300D [ERBE, Tubingen, Germany] or ESG 100 [Olympus]). As the injection solution, we used a 1:1 mixture of $0.4 \%$ sodium hyaluronate (MucoUp ${ }^{\circledR}$; Johnson \& Johnson, New Brunswick, NJ, USA) and $10 \%$ glycerin solution plus a minute amount of indigo carmine. ${ }^{1-4}$ We performed submucosal layer dissection using WLI and switched to DRI when exposed blood vessels were detected in the artificial ulcer or when unexpected bleeding occurred. We performed preventive coagulation for all detected vessels using hemostatic forceps, through soft coagulation (effect 4,60 W) with an electrosurgical generator (VIO300D; ERBE). ${ }^{5}$ Video processors (EVIS LUCERA ELITTE system; Olympus) were used for recording the ESD procedure. The moment bleeding from blood vessels in the submucosal layer occurred during the dissection, we switched from WLI to DRI.

\section{Evaluation in still pictures}

First, we compared the visibility of blood vessels in the submucosal layer in images captured using DRI with the corresponding images captured using WLI. A total of 34 images selected from the 20 cases were assessed. Magnified images of the blood vessels were obtained from the same distance and

angle as those used to obtain nonmagnified images. One or more images from all cases were randomly selected.

\section{Evaluation in videos}

We compared the differences in the visibility of bleeding points between the DRI images and the corresponding WLI images in the videos. All available scenes were extracted from the recorded videos of the 20 patients, and 1 scene from the videos of each patient was selected randomly. These randomly chosen scenes from the 20 patients were used for examination. Videos of the bleeding point were obtained from the same distance and angle as those for the nonmagnified images in WLI and DRI. A total of 5 experts who had performed $>50$ gastric ESD procedures compared the DRI images and videos with the corresponding WLI images and videos. The endoscopic images were presented to each of the experts in a random order for comparing WLI and DRI. They scored the images from DRI relative to the corresponding images from WLI for visibility of blood vessels according to the following scale: +2 (improved visibility), +1 (slightly improved visibility), 0 (visibility equivalent to that of WLI), -1 (worsened visibility), and -2 (visibility lost) (Table 1). ${ }^{6,7}$ The total scores of the 5 endoscopists were calculated. In this study, the highest possible score was +10 and the lowest was -10 . A score $\geq+5$ was defined as improved; between +4 and -4 , as equivalent; and $\leq-5$, as decreased, as previously reported. ${ }^{6-8}$

Next, we investigated the clinicopathological features of

Table 1. Visibility of Dual Red Imaging Corresponding to White Light Imaging

\begin{tabular}{lcc}
\hline Visibility & $\begin{array}{c}\text { Visibility of } \\
\text { vessels } \\
(\boldsymbol{n}=\mathbf{3 4})\end{array}$ & $\begin{array}{c}\text { Detection of the } \\
\text { bleeding point } \\
(\boldsymbol{n}=\mathbf{2 0})\end{array}$ \\
\hline Improved & $56 \%$ & $55 \%$ \\
& $(19 / 34)$ & $(11 / 20)$ \\
No change & $44 \%$ & $45 \%$ \\
& $(15 / 34)$ & $(9 / 20)$ \\
Decreased & $0 \%$ & $0 \%$ \\
& $(0 / 34)$ & $(0 / 20)$ \\
\hline
\end{tabular}
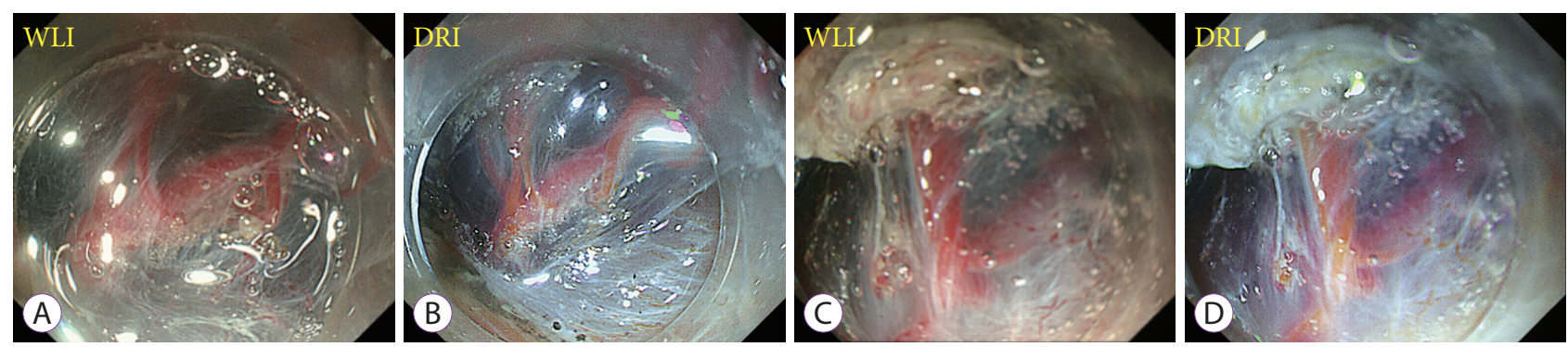

Fig. 1. Representative cases showing the usefulness of dual red imaging (DRI) in improving the visibility of blood vessels. Images from white light imaging (WLI) (A, C) and their corresponding DRI images $(B, D)$. DRI enhanced the color tone of arteries and veins and improved visibility. 
the cases in the DRI-improved group and the other groups. To examine the useful conditions for DRI, we compared the clinicopathological features of the DRI-improved group with those of the DRI-equivalent group. We compared the tumor diameter $(\mathrm{mm})$, diameter of the resected specimen $(\mathrm{mm})$, tumor location (upper, middle, or lower body of the stomach), operation time (min), fibrosis of the submucosal layer (F0, F1, F2), incidence of complications (perforation, delayed
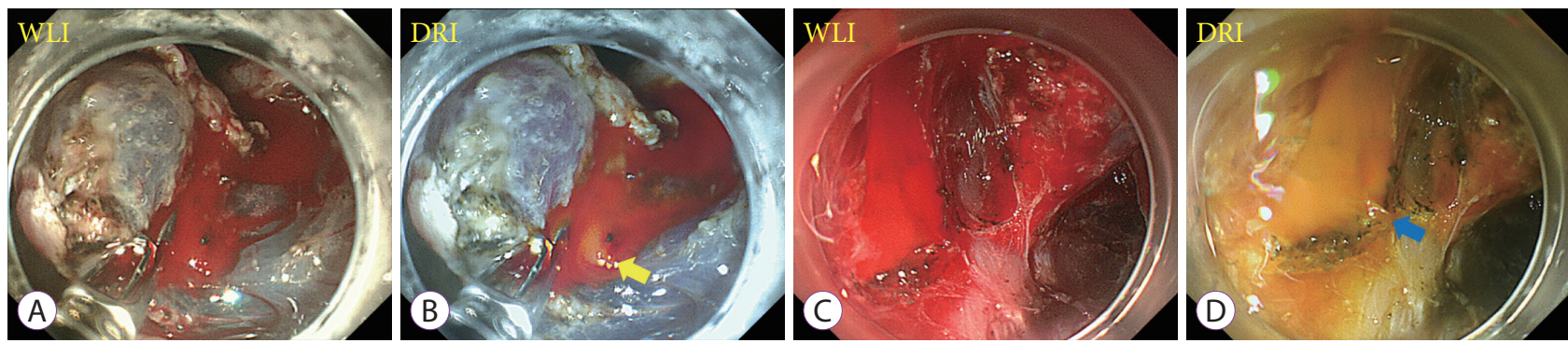

Fig. 2. Representative cases showing the usefulness of dual red imaging (DRI) in improving the visibility of bleeding points. Images from white light imaging (WLI) (A, C) and their corresponding DRI images (B, D). DRI emphasized the blood flow from the bleeding points in orange color, which made it easier to accurately detect the bleeding points (yellow and blue arrows).

Table 2. Clinicopathological Features of the Visibility of the Bleeding Point Improved Group and Equivalent Group in Dual Red Imaging

\begin{tabular}{|c|c|c|c|}
\hline \multirow{2}{*}{ Variables } & \multicolumn{2}{|c|}{ Visibility of the bleeding points } & \multirow{2}{*}{$p$-value } \\
\hline & Improved $(n=11)$ & Equivalent $(n=9)$ & \\
\hline Tumor diameter (mm) & & & $\mathrm{N} / \mathrm{S}$ \\
\hline$<20$ & $8(73)$ & $7(78)$ & \\
\hline$\geq 20$ & $3(27)$ & $2(22)$ & \\
\hline Resected specimen $($ mean $\pm \mathrm{SD}, \mathrm{mm})$ & $45 \pm 23$ & $43 \pm 22$ & $\mathrm{~N} / \mathrm{S}$ \\
\hline Location & & & $\mathrm{N} / \mathrm{S}$ \\
\hline $\mathrm{U}$ & $3(27)$ & $7(64)$ & \\
\hline $\mathrm{M}$ & $6(55)$ & $1(18)$ & \\
\hline $\mathrm{L}$ & $2(18)$ & $1(18)$ & \\
\hline Operation time (min) & $52 \pm 35$ & $48 \pm 32$ & $\mathrm{~N} / \mathrm{S}$ \\
\hline Fibrosis & & & $\mathrm{N} / \mathrm{S}$ \\
\hline F0 & $6(55)$ & $6(67)$ & \\
\hline $\mathrm{F} 1+\mathrm{F} 2$ & $5(45)$ & $3(33)$ & \\
\hline Blood pooling & & & 0.02 \\
\hline Present & $9(82)$ & $2(22)$ & \\
\hline Absent & $2(18)$ & $7(78)$ & \\
\hline Bleeding type & & & 0.02 \\
\hline Oozing & $9(82)$ & $2(22)$ & \\
\hline Spurting & $2(18)$ & $7(78)$ & \\
\hline Number of bleeding points & & & $\mathrm{N} / \mathrm{S}$ \\
\hline Single & $4(36)$ & $5(56)$ & \\
\hline Multiple & $7(64)$ & $4(44)$ & \\
\hline Number of coaglation & $1.9 \pm 0.5$ & $2.5 \pm 0.7$ & 0.04 \\
\hline Complications & & & $\mathrm{N} / \mathrm{S}$ \\
\hline Delayed bleeding & $0(0)$ & $0(0)$ & \\
\hline Perforation & $0(0)$ & $0(0)$ & \\
\hline
\end{tabular}

L, lower third of the stomach; M, middle third of the stomach; N/S, not significant; SD, standard deviation; $\mathrm{U}$, upper third of the stomach. 
bleeding), blood pooling around the bleeding point (present vs. absent), bleeding type (oozing vs. spurting), number of bleeding vessels (single, multiple), and number of coagulations performed for complete hemostasis. The presence of submucosal fibrosis was evaluated with WLI. Endoscopic findings were classified as previously reported ${ }^{3}$ and were based on observations at the time of injection of sodium hyaluronate with indigo carmine: $\mathrm{F} 0$, no fibrosis, which manifests as a blue transparent layer; F1, mild fibrosis, which appears as a white web-like structure in the blue submucosal layer; and F2, severe fibrosis, which appears as a white muscular structure without a blue transparent layer in the submucosal layer.

\section{Statistical analysis}

Quantitative data are expressed as means and standard deviations or percentages. Clinicopathological features were analyzed using the chi-square test. A value $p<0.05$ was considered statistically significant. Statistical analysis was performed using JMP ${ }^{\circledR}$ software (SAS International Inc., Cary, NC, USA).

\section{RESULTS}

The findings of blood vessels and bleeding points on DRI and WLI are shown in Table 1 . The visibility of blood vessels was improved in 56\% (19/34) of the cases and equivalent visibility was observed in $44 \%$ (15/34) of the cases (Fig. 1). There were no cases with decreased visibility of blood vessels. The visibility of bleeding points was improved in 55\% (11/20) of the cases and equivalent visibility was observed in $45 \%(9 / 20)$ of the cases (Fig. 2). There were no cases with decreased visibility of bleeding points.

The clinicopathological features of the cases in the DRI-improved and DRI-equivalent groups are shown in Table 2. With respect to tumor diameter, resected specimen diameter, tumor location, operation time, and submucosal layer fibrosis, no significant differences were found between the groups. There were no complications in any of the cases. The extent of fibrosis did not affect the usefulness of DRI. Pooling of blood around the bleeding point was seen in $82 \%$ (9/11) of the improved group and $22 \%(2 / 11)$ of the equivalent group $(p=0.02)$. With respect to the type of bleeding, oozing was seen in $82 \%$ $(9 / 11)$ of the improved group and $22 \%(2 / 11)$ of the equivalent group $(p=0.02$ ). Multiple bleeding points were detected in $64 \%$ (7/11) of the improved group and $44 \%$ (4/9) of the equivalent group. The number of coagulations needed for complete hemostasis was $1.9 \pm 0.5$ in the improved group and $2.5 \pm 0.7$ in the equivalent group $(p=0.04)$.

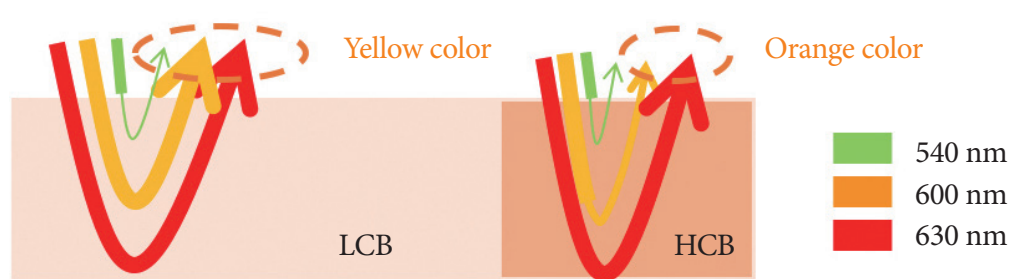

(A)

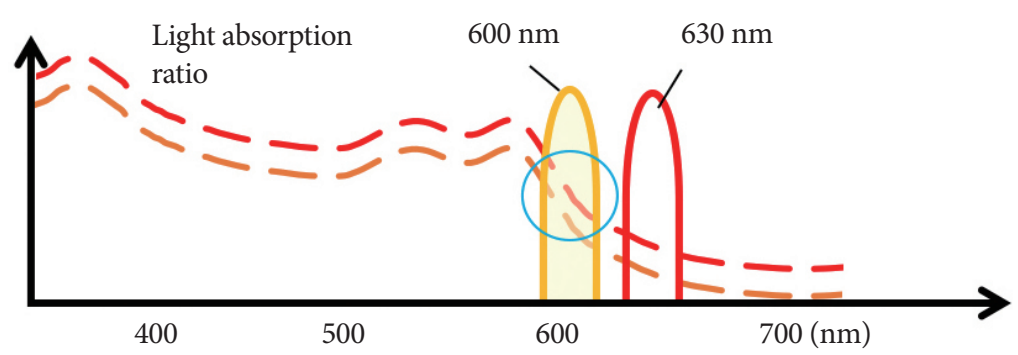

Light absorption characteristics

(B)

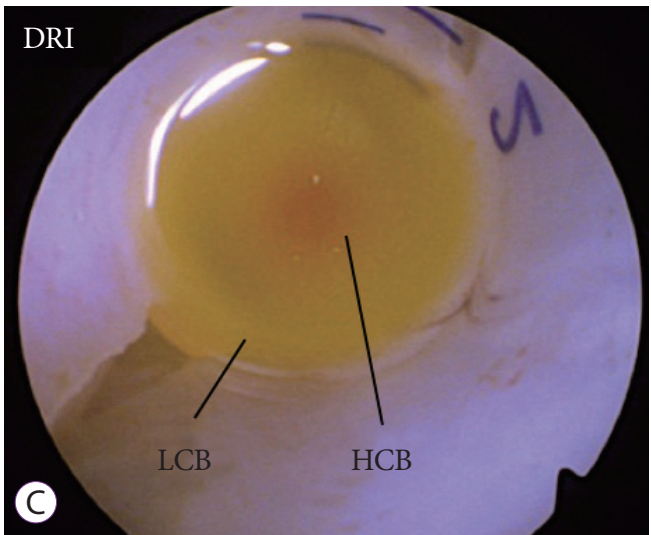

Fig. 3. Mechanism of the dual red imaging (DRI) system. (A) In low concentration of blood, most of the 600-nm wavelength (orange color) is not attenuated, whereas the same wavelength is attenuated in the presence of a high concentration of blood. (B) Because yellow light of $600 \mathrm{~nm}$ is attenuated more in high-concentrated blood than in low-concentrated blood, the reflected image becomes reddish and contrast occurs. DRI was used at the 2 different blood concentrations. (C) A small amount of high-density blood was injected backward into the low-concentrated blood. DRI can distinguish between the different concentrations of blood. HCB, high concentration blood; LCB, low concentration blood. 


\section{DISCUSSION}

Our data revealed that the visibility of blood vessels and bleeding points was improved with the use of DRI during gastric ESD. DRI was found to be useful in improving visibility in cases showing blood pooling around the bleeding point, oozing bleeding type, and multiple bleeding points. Moreover, the number of coagulations needed for complete hemostasis was significantly smaller in the improved group. On the other hand, in the equivalent group, spurting bleeding and the lack of blood pooling was detected in many cases. In cases in which the bleeding point was clearly detected by WLI, further improvement was not found with the use of DRI. Even if those assessments were correct, the decreased number of coagulations needed for complete hemostasis for each bleeding point in the improved group may further imply the usefulness of DRI. However, the effects of DRI in shortening the procedure time and reducing complications were not proven in our study.

In our previous study, we reported that when there was poor control of bleeding during ESD, the risk of post-ESD bleeding was higher. ${ }^{10}$ It has been reported that in difficult cases of ESD, endoscopic hemostasis becomes an obstacle, and the operation time tends to be longer. ${ }^{11}$ To avoid delayed perforation, the bleeding points should be grasped precisely with hemostatic forceps. We have reported that an operation time of $\geq 75 \mathrm{~min}$ is an independent risk factor for bleeding after ESD; hence, shortening the time of hemostasis is important. ${ }^{12}$ Excessive hemostasis may require special measures such as transfusion or emergency surgery. ${ }^{13}$ Yahagi et al. reported that DRI improved the visibility of bleeding points and shortened the time needed for hemostasis during gastric and colorectal ESD. ${ }^{14}$ Although shortening of the procedure time was not demonstrated in the present study, we believe that the usefulness of DRI may become clearer if focus is placed on high-risk cases for hemostasis during gastric ESD in the near future.

We previously reported that DRI improved the visibility of arteries and the submucosal layer in colorectal ESD. ${ }^{6}$ Because DRI makes hemostasis easier in many cases, DRI greatly improves the visibility of blood vessels. ${ }^{6,14}$ The orange color of the bloodstream makes it easy to assess the bleeding point. The usefulness of DRI in endoscopic hemostasis for spurting duodenal bleeding has also been reported. Tanaka et al. reported that DRI improved the visibility of spurting bleeding by displaying it as a yellow flow in difficult conditions with persistent hemorrhage. ${ }^{15}$ DRI enhances the differences in blood concentrations. In low blood concentration, most waves with a wavelength of $600 \mathrm{~nm}$ are not attenuated, whereas they are attenuated in the presence of a high concentration of blood. Because yellow light of 600-nm wavelength becomes highly attenuated with more highly concentrated blood than with low-concentrated blood, the reflected image becomes reddish and a color contrast occurs (Fig. 3). This was the mechanism responsible for the detection of bleeding points in cases in which there was blood pooling. In DRI, the active bleeding point is depicted in orange because of the high concentration of blood. In contrast, blood pooling around the bleeding point is depicted in yellow because blood concentration decreases when mixed with gastric mucus. We considered DRI to be especially effective in cases with blood pooling around the bleeding vessels in endoscopic hemostasis.

Naganuma et al. reported that DRI was useful in predicting the prognosis of ulcerative colitis in patients with mild-to-moderate inflammation because it could assess the deep vessels and DRI scores correlated with the severity of inflammation. ${ }^{16}$ Furuichi et al. reported that DRI was useful in predicting the depth of esophageal varices and led to an increase in the endoscopic injection sclerotherapy success rate owing to the accurate adjustment of the puncture needle. ${ }^{17,18}$ Tanaka et al. reported that DRI was useful in maintaining clear visibility during colorectal ESD, especially with submucosal fatty tissue. ${ }^{19}$ Thus, the usefulness of DRI is expected to be clarified in the near future.

This study has some limitations. First, it was a retrospective study and involved a review of endoscopic images and videos. Second, the number of cases was relatively small. In addition, there was some selection bias in the enrolled cases because the number of procedures and the uses of DRI were limited because of the use of a prototype scope. Thus, a prospective study should be performed to investigate the usefulness of DRI.

In conclusion, during gastric ESD, DRI makes it possible to visualize the bleeding points, especially under difficult conditions such as blood pooling and oozing bleeding. DRI is helpful in gastric ESD.

\section{Conflicts of Interest}

The authors have no financial conflicts of interest.

\section{Author Contributions}

Conceptualization: Shiro Oka

Data curation: Naoki Yorita, Naoko Nagasaki, Kazutaka Kuroki Formal analysis: NY, SO

Investigation: NY, SO, Shinji Tanaka, Takahiro Kotachi, NN, Kosaku Hata, KK, Kazuhiko Masuda, Mio Kurihara, Mariko Kiso, Tomoyuki Boda, Masanori Ito

Methodology: NY, SO, ST, TK, NN, KH, KK, KM, MKu, MKi, TB, MI

Project administration: SO, ST, Kazuaki Chayama

Resources: NY, SO

Software: SO

Supervision: SO, ST

Validation: NY, SO, ST

Visualization: NY, SO, ST 
Writing-original draft: NY, SO

Writing-review\&editing: NY, SO, ST

\section{REFERENCES}

1. Oka S, Tanaka S, Kaneko I, et al. Advantage of endoscopic submucosal dissection compared with EMR for early gastric cancer. Gastrointest Endosc 2006;64:877-883.

2. Oka S, Tanaka S, Kaneko I, et al. Endoscopic submucosal dissection for residual/local recurrence of early gastric cancer after endoscopic mucosal resection. Endoscopy 2006;38:996-1000.

3. Higashimaya M, Oka S, Tanaka S, et al. Outcome of endoscopic submucosal dissection for gastric neoplasm in relationship to endoscopic classification of submucosal fibrosis. Gastric Cancer 2013;16:404-410.

4. Sanomura Y, Oka S, Tanaka S, et al. Clinical validity of endoscopic submucosal dissection for submucosal invasive gastric cancer: a single-center study. Gastric Cancer 2012;15:97-105.

5. Nagata S, Kimura S, Ogoshi H, Hidaka T. Endoscopic hemostasis of gastric ulcer bleeding by hemostatic forceps coagulation. Dig Endosc 2010;22 Suppl 1:S22-S25.

6. Ninomiya Y, Oka S, Tanaka S, et al. Clinical impact of dual red imaging in colorectal endoscopic submucosal dissection: a pilot study. Therap Adv Gastroenterol 2016;9:679-683.

7. Imagawa $\mathrm{H}$, Oka S, Tanaka S, et al. Improved visibility of lesions of the small intestine via capsule endoscopy with computed virtual chromoendoscopy. Gastrointest Endosc 2011;73:299-306.

8. Yoshifuku Y, Sanomura Y, Oka S, et al. Evaluation of the visibility of early gastric cancer using linked color imaging and blue laser imaging. BMC Gastroenterol 2017;17:150.

9. Matsumoto A, Tanaka S, Oba S, et al. Outcome of endoscopic submucosal dissection for colorectal tumors accompanied by fibrosis. Scand J
Gastroenterol 2010;45:1329-1337.

10. Higashiyama M, Oka S, Tanaka S, et al. Risk factors for bleeding after endoscopic submucosal dissection of gastric epithelial neoplasm. Dig Endosc 2011;23:290-295.

11. Jeon SW, Jung MK, Cho CM, et al. Predictors of immediate bleeding during endoscopic submucosal dissection in gastric lesions. Surg Endosc 2009;23:1974-1979.

12. Sanomura Y, Oka S, Tanaka S, et al. Taking warfarin with heparin replacement and direct oral anticoagulant is a risk factor for bleeding after endoscopic submucosal dissection for early gastric cancer. Digestion 2018;97:240-249.

13. Tajiri H, Kitano S. Complications associated with endoscopic mucosal resection: definition of bleeding that can be viewed as accidental. Dig Endosc 2004;16(Suppl 1):S134-S136.

14. Yahagi N, Horii J, Goto O, et al. Dual red imaging; a new endoscopic imaging technology for clear visualization of bleeding points in endoscopic submucosal dissection. Gastrointest Endosc 2014;79(5 Suppl):AB464.

15. Tanaka H, Oka S, Tanaka S. Endoscopic hemostasis for spurting duodenal bleeding using dual red imaging. Dig Endosc 2017;29:816-817.

16. Naganuma M, Yahagi N, Bessho R, et al. Evaluation of the severity of ulcerative colitis using endoscopic dual red imaging targeting deep vessels. Endosc Int Open 2017;5:E76-E82.

17. Furuichi Y, Gotoda T, Kasai Y, et al. Role of dual red imaging to guide intravariceal sclerotherapy injection of esophageal varices (with videos). Gastrointest Endosc 2018;87:360-369.

18. Furuichi Y, Gotoda T, Moriyasu F, et al. Dual red imaging (novel advanced endoscopy) can increase visibility and can predict the depth in diagnosing esophageal varices. J Gastroenterol 2017;52:568-576.

19. Tanaka H, Oka S, Tanaka S, et al. Dual red imaging maintains clear visibility during colorectal endoscopic submucosal dissection. Dig Dis Sci 2019;64:224-231. 